\title{
Treinamento auditivo computadorizado em idosos protetizados pelo Sistema Único de Saúde
}

\section{Computer-based auditory training for elderly with hearing aids}

\author{
provided by the Health Care System
}

\author{
Thais de Sena Teixeira', Maria Inês Dornelles da Costa-Ferreira'
}

\begin{abstract}
RESUMO
Objetivo: Verificar a efetividade de um programa de treinamento auditivo computadorizado em idosos protetizados. Métodos: $\mathrm{O}$ estudo foi realizado com 72 idosos, entre 60 e 89 anos de idade, média de 74 anos $( \pm 7,8)$, protetizados em um centro de saúde. Os participantes foram divididos em grupo experimental, 48 (66,7\%) e grupo controle, 24 (33,3\%). Todos foram submetidos aos testes comportamentais (Fala no Ruído, Random Gap Detection Test e Dicótico de Dígitos) e ao questionário de restrição de participação Hearing Handicap Inventory for the Elderly - Screening Version. O grupo experimental foi avaliado antes e após o treinamento auditivo (quatro sessões) e o grupo controle, na entrega do aparelho de amplificação sonora individual e 40 dias após. O treinamento auditivo neurocognitivo foi elaborado conforme as necessidades de cada participante, com a utilização de recursos variados. Resultados: Ao comparar o desempenho nos testes comportamentais e no questionário de restrição de participação, nos dois momentos de avaliação, identificou-se diferença estatística para ambos os grupos. O coeficiente delta de cada variável permitiu a análise da variação entre a pré-avaliação e a pós-avaliação e constatou diferenças estatisticamente significativas para os testes comportamentais e não significativas para o questionário de restrição de participação. Conclusão: O programa de treinamento auditivo computadorizado se mostrou efetivo, indicando melhora das habilidades auditivas alteradas. O questionário não refletiu os efeitos do treinamento auditivo.
\end{abstract}

Palavras-chave: Perda auditiva; Auxiliares de audição; Reabilitação; Envelhecimento; Serviços de saúde

\begin{abstract}
Purpose: To check effectiveness of a computer-based auditory training program for elderly citizens with hearing aids. Methods: The study was conducted with 72 senior patients, between 60 and 89 years old, with an average age of 74 years $( \pm 7.8)$, all users of hearing aids fitted in a health center. The patients were divided in an experimental group, 48 users (66.7\%), and a control group, $24(33.3 \%)$. They were all part of the same behavioral tests (speech in noise, random gap detection and dichotic digit test) and also the Hearing Handicap Inventory for the Elderly - Screening Version restriction questionnaire. The experimental group was tested before and after the auditory training (four sessions), while the control group was tested at the delivery of the individual hearing aids and 40 days later. The neurocognitive auditory training was customized to each participant with the use of varied resources. Results: After comparing the behavioral tests results and the restriction questionnaire answers, in both evaluation moments, we noticed a statistical advantage for both groups. The analysis using a delta coefficient of each variable allowed us to visualize the variation from pre-evaluation to post-evaluation. The result showed a significant increase in the auditory processing tests and a not significant increase in the restriction questionnaire. Conclusion: The computer-based auditory training program was effective, producing improvements in the hearing abilities. The restriction questionnaire did not reflect the effects of the auditory training.
\end{abstract}

Keywords: Hearing loss; Hearing aids; Rehabilitation; Aging; Health services

\footnotetext{
Trabalho realizado no Centro de Saúde Clélia Manfro na Unidade Auditório - Caxias do Sul (RS), Brasil.

${ }^{1}$ Faculdade Nossa Senhora de Fátima - Caxias do Sul (RS), Brasil.

Conflito de interesses: Não.

Contribuição dos autores: TST foi responsável pela escrita do artigo, análise e interpretação dos dados; MIDCF foi responsável pela concepção e delineamento do estudo, análise e interpretação dos dados, revisão do artigo.

Financiamento: Fundação de Amparo à Pesquisa do Rio Grande do Sul (FAPERGS), processo número 1139-2551/13-1.

Autor correspondente: Maria Inês Dornelles da Costa-Ferreira. E-mail: costa.ferreira@terra.com.br

Recebido: Setembro 07, 2016; Aceito: Dezembro 11, 2016
} 


\section{INTRODUÇÃO}

A audição desempenha um importante papel na sociedade ${ }^{(1)}$. Dentre as alterações sensoriais que acompanham o processo de envelhecimento, a deficiência auditiva é uma das mais incapacitantes, pois a deterioração do sistema auditivo causa deficit na compreensão da fala, gerando dificuldades de inserção social $^{(2,3)}$.

Além da perda auditiva, outra questão que deve ser ressaltada é a desvantagem auditiva (handicap), que também está relacionada a outros aspectos que não são específicos da audição, implicando a limitação do indivíduo ao desempenhar suas atividades ${ }^{(4)}$. O processo de reabilitação auditiva busca minimizar as desvantagens do indivíduo com perda auditiva e, a partir disso, os idosos se tornam candidatos ao uso do aparelho de amplificação sonora individual (AASI) ${ }^{(3)}$. Para que o processo de seleção, indicação e adaptação do AASI seja eficaz, é necessário que o indivíduo faça uso efetivo deste dispositivo e, para que isso ocorra, precisa estar satisfeito com os resultados da adaptação ${ }^{(5)}$.

Por este motivo, torna-se importante conhecer as associações entre a deficiência auditiva e o transtorno do processamento auditivo, pois é comum observar idosos que possuem integridade auditiva periférica e/ou idosos protetizados com ganho funcional adequado para a perda auditiva, mas que persistem com dificuldades de compreensão de fala, principalmente na presença de ruído. Estudos recentes têm evidenciado que tais dificuldades podem ser relacionadas às de processamento auditivo ${ }^{(6)}$.

O processamento auditivo é a maneira como o sistema auditivo recebe, organiza e interpreta os sons ouvidos. Consiste numa função cerebral fundamental à identificação, localização, discriminação e memorização dos estímulos acústicos, sendo um conjunto de habilidades específicas das quais o indivíduo depende para compreender o que ouve $\mathrm{e}^{(7,8)}$.

A avaliação do processamento auditivo verifica como o indivíduo recebe as informações acústicas e pode auxiliar na identificação dos aspectos subjacentes a algumas dificuldades, principalmente em idosos que, frequentemente, relatam queixas de compreensão ${ }^{(9)}$. Além do declínio da função auditiva, algumas funções cognitivas diminuem naturalmente com a idade. Estudos indicaram que as habilidades auditivas centrais estariam relacionadas às funções cognitivas e, por este motivo, os testes comportamentais do processamento auditivo verificam as habilidades auditivas, cujos resultados podem refletir os efeitos do envelhecimento ${ }^{(1,10,11)}$.

O treinamento auditivo é uma das estratégias aplicadas para a reabilitação auditiva em usuários de AASI com alterações nas habilidades avaliadas e tem como fundamento a estimulação auditiva, com o objetivo de maximizar os efeitos da plasticidade do sistema nervoso central ${ }^{(12,13)}$. Assim, o treinamento auditivo computadorizado permite o controle de dados e a hierarquia das atividades e vem sendo aplicado para fortalecer as sinapses e promover a formação de novos engramas, melhorando, assim, as habilidades auditivas. Softwares especializados são utilizados na intervenção, sendo uma prática inovadora ${ }^{(13)}$.

Resumidamente, o treinamento auditivo estimula as vias auditivas centrais para que o indivíduo se torne um ouvinte mais ativo e hábil ${ }^{(12,13)}$, porém, faz-se necessário identificar as variáveis que interferem nesse processo, como o benefício do AASI e o desempenho cognitivo do indivíduo.
Com base nas informações mencionadas e na escassez de estudos que abordem o treinamento auditivo realizado no Sistema Único de Saúde (SUS), que preconiza apenas quatro sessões, justifica-se a realização desta pesquisa, que poderá contribuir para a verificação da importância do uso efetivo do AASI, bem como para a elaboração do treinamento auditivo, tendo como finalidade minimizar as alterações nas habilidades auditivas causadas pela privação sensorial. Sendo assim, o presente estudo teve como objetivo verificar a efetividade de um programa de treinamento auditivo computadorizado em idosos protetizados pelo SUS, por meio das medidas de processamento auditivo e questionário de restrição de participação.

\section{MÉTODOS}

A pesquisa foi realizada Centro de Saúde Clélia Manfro Unidade Auditiva e recebeu aprovação do Comitê de Ética em Pesquisa da Faculdade Nossa Senhora de Fátima, sob o protocolo 918.462/2014. Caracteriza-se por ser um estudo longitudinal, com intervenção, retrospectivo e prospectivo, observacional, contemporâneo e individual.

Inicialmente, o responsável pelo Centro de Saúde Auditiva assinou o Termo de Conhecimento Institucional (TCI). Na sequência, os idosos do programa de saúde auditiva que se encaixaram nos critérios de inclusão foram convidados a participar do projeto e, após a aceitação, assinaram o Termo de Consentimento Livre e Esclarecido (TCLE), que foi lido em conjunto.

A amostra foi composta por sujeitos de ambos os sexos, com idade mínima de 60 anos e máxima de 89 anos, diagnosticados com perda auditiva neurossensorial de grau leve a moderadamente severo, que foram encaminhados ao setor de prótese auditiva no período de abril de 2014 a dezembro de 2015.

Foram incluídos pacientes com perda auditiva neurossensorial bilateral, simétrica ${ }^{(14)}$, de grau leve a moderadamente severo ${ }^{(15)}$, encaminhados para seleção, indicação e adaptação de AASI.

Participaram do grupo experimental os idosos que compareceram a todas as etapas da pesquisa, ou seja, na aplicação do questionário Hearing Handicap Inventory for the Elderly - Screening Version (HHIE-S), na realização dos testes comportamentais após a entrega do AASI, nas quatro sessões de treinamento auditivo preconizadas pelo SUS e, após o término do treinamento, para reavaliação e nova aplicação do questionário HHIE-S. Participaram do grupo controle os idosos que compareceram à realização dos testes comportamentais e do HHIE-S após a entrega do AASI e após 40 dias de uso, para reavaliação. Todos os idosos, de ambos os grupos, utilizavam AASI bilateral do tipo mini retroauricular, com receptor no canal.

Foram excluídos do estudo os pacientes que não compareceram a qualquer uma das etapas da pesquisa, que foram diagnosticados com perdas auditivas de grau profundo e/ou perdas auditivas condutivas ou mistas, pacientes que apresentaram história clínica de alterações da orelha externa e/ou média, ou que apresentaram alterações auditivas às medidas de imitância acústica e pacientes antigos, usuários de AASI. Igualmente foram excluídos aqueles que apresentaram comprometimentos cognitivos severos ou alterações neurológicas que impediram a realização dos protocolos. Também foram realizados os seguintes testes: Escala de Depressão Geriátrica (EDG), subteste de reconhecimento de palavras da Escala de Avaliação da Doença de Alzheimer 
Cognitiva (ADAS-Cog) e teste de fluência verbal. Os indivíduos que não conseguiram realizá-los foram excluídos do estudo.

$\mathrm{Na}$ data em que os pacientes de ambos os grupos compareceram para a entrega do AASI, foi verificado o interesse em participar da pesquisa e, após a concordância, foi realizada a leitura e assinatura TCLE. Na mesma data, foi aplicado o questionário HHIE-S e agendado o próximo encontro, quando, então, foram realizados os testes comportamentais.

O questionário HHIE-S é uma versão de triagem, com dez itens retirados do Hearing Handicap Inventory for the Elderly - HHIE, que foi desenvolvido para avaliar o impacto da perda auditiva no ajuste emocional e social do paciente idoso. O grau de restrição de participação é determinado conforme os critérios de pontuação das respostas. Quanto maior o índice, maior é a percepção do indivíduo em relação ao seu handicap, ou seja, maiores são as dificuldades causadas pela perda auditiva ${ }^{(16)}$.

Os testes comportamentais escolhidos para o presente estudo foram: teste de Fala no Ruído, Random Gap Detection Test (RGDT) convencional ou expandido (somente realizado caso o paciente não conseguisse responder ao convencional) e Dicótico de Dígitos. O teste Fala no Ruído foi escolhido em função da principal queixa dos idosos, ou seja, a dificuldade de compreender a fala em ambientes competitivos. Também se optou pelo teste RGDT por ser uma medida de resolução temporal mais breve, se comparado a outro teste disponível. Já o teste Dicótico de Dígitos foi proposto para avaliar a binauralidade para sons verbais com menor carga linguística, se comparado a outra medida. Os pacientes do grupo experimental retornaram após a realização dos testes comportamentais, para as quatro sessões de treinamento auditivo e, após a finalização, foi realizada a reavaliação, em que foram utilizados os mesmos testes. Os pacientes do grupo controle retornaram após 40 dias de uso do AASI (tempo em que o grupo experimental realizou o treinamento auditivo), para a reavaliação.

Os testes foram realizados com o participante em cabine acústica, utilizando fones supra-aurais TDH-39, sem o AASI. Foram gravados em $\mathrm{CD}$ e aplicados por meio de um audiômetro de dois canais (AC 33, da marca Interacoustics ${ }^{\circledR}$ ), acoplado a um notebook (Inspiron 142620, da marca Dell ${ }^{\circledR}$ ). Todos os testes foram realizados numa intensidade de $40 \mathrm{dBNS}$ acima da média audiométrica das frequências de $500 \mathrm{~Hz}$ a $2000 \mathrm{~Hz}$ do indivíduo. Convém ressaltar que a média tritonal máxima encontrada foi de $60 \mathrm{~dB} \mathrm{NA}^{(15)}$. Todos os equipamentos encontravam-se calibrados no período em que as avaliações foram realizadas.

Primeiramente, foi realizado o teste Fala no Ruído, que consiste na apresentação simultânea de 25 monossílabos e de ruído branco, na mesma orelha. É solicitado ao paciente que repita as palavras ouvidas, ignorando o ruído ipsilateral. A relação sinal/ruído utilizada foi de $0 \mathrm{~dB}$, visto que a amostra era composta por idosos com perda auditiva periférica ${ }^{(17)}$. Este teste é destinado à avaliação da habilidade auditiva de fechamento auditivo.

Na sequência, aplicou-se o teste RGDT, em que são apresentados tons puros pareados com pequenos intervalos de silêncio. Caso o indivíduo não respondesse ao RGDT convencional, cujos intervalos de tempo são menores, variando de 0 a 40 ms, aplicava-se o RGDT expandido, cujos intervalos de tempo variam de 50 a 300 ms, para facilitar a identificação dos intervalos.

A orientação ao sujeito em avaliação é que responda verbalmente ou que aponte se ouviu um ou dois estímulos. O limiar de detecção de intervalo é considerado como sendo o menor intervalo a partir do qual o indivíduo passou a identificar consistentemente a ocorrência de dois estímulos. $\mathrm{O}$ valor de referência para este teste são as respostas compreendidas entre $2 \mathrm{~ms}$ e $10 \mathrm{~ms}^{(18)}$. O teste avalia a resolução temporal, sendo definida como a habilidade do sistema auditivo em detectar mudanças rápidas no estímulo sonoro, ou o menor intervalo de tempo necessário para discriminar dois estímulos acústicos.

Por fim, realizou-se o teste Dicótico de Dígitos - etapa de integração binaural, que consiste em uma gravação com 20 pares de dígitos. Foram apresentados aos participantes quatro dígitos de forma dicótica, simultaneamente, sendo dois numa orelha e dois na outra. A resposta esperada era a repetição oral dos dígitos apresentados ${ }^{(17)}$.

Para o treinamento auditivo computadorizado, foram utilizados os softwares Escuta Ativa ${ }^{\circledR}$, Duo Training ${ }^{\circledR}$, Memo Training ${ }^{\circledR}$, Pedro no Acampamento ${ }^{\circledR}$ e o CD de áudio Escutando com Interferentes ${ }^{\circledR}$. Foram realizadas quatro sessões de treinamento auditivo, conforme a proposição da Portaria SAS/MS n ${ }^{\circ} 587$, de 07 de outubro de $2004^{(19)}$, sendo uma sessão por semana, com duração de uma hora cada. O treinamento auditivo foi realizado com fones supra-aurais da marca Bose ${ }^{\circledR}$, acoplados ao computador. Por ser um acessório leve, não ocluiu a orelha a ponto de gerar microfonia, permitindo a realização do treinamento em usuários de AASI do tipo mini retroauricular.

As atividades foram planejadas de acordo com as necessidades de cada paciente, observadas por meio da anamnese, da avaliação audiológica básica, dos resultados obtidos no questionário de restrição de participação (HHIE-S) e nos testes comportamentais realizados anteriormente à adaptação do AASI: teste de fluência verbal (medida de função executiva), subteste de reconhecimento de palavras da Escala de Avaliação da Doença de Alzheimer Cognitiva (ADAS-Cog), que avalia memória de curto prazo e Escala de Depressão Geriátrica (EDG). Os dados dos três últimos instrumentos não serão apresentados nos resultados deste trabalho, dada a limitação de espaço do artigo, mas foram considerados na proposição do treinamento auditivo. As atividades utilizadas nas quatro sessões de treinamento auditivo basearam-se na estimulação das habilidades auditivas que se encontravam alteradas nos participantes com perda auditiva, incluindo o treinamento das funções cognitivas de atenção, memória, linguagem e função executiva.

As habilidades que se observaram alteradas nos idosos avaliados, por meio do teste Dicótico de Dígitos, foram estimuladas a partir das atividades "Bem na Mira" e "Esquerda/ Direita" do software Escuta Ativa ${ }^{\circledR}$ e com as listas de palavras "Oposições Simples" e "Pares em Oposição" do software Duo Training $^{\circledR}$, visando melhorar a binauralidade. A habilidade de discriminação no silêncio e no ruído, que se observou alterada nos idosos avaliados, por meio do teste Fala no Ruído, foi estimulada a partir da atividade "Qual Som Ouviu" do software Escuta Ativa ${ }^{\circledR}$ e das faixas do CD de áudio Escutando com Interferentes $^{\circledR}$, visando melhorar a discriminação auditiva.

As atividades do software Pedro no Acampamento ${ }^{\circledR}$ e do software Memo Training ${ }^{\circledR}$ foram trabalhadas apenas com os participantes que apresentaram, nas avaliações realizadas, indícios de alterações nas funções cognitivas de atenção, memória, linguagem e/ou função executiva. As tarefas mais utilizadas foram: "Travessia no Rio", "Baú do Tesouro" e "Refeitório".

Quando o teste de fluência verbal era alterado, foram propostas as atividades denominadas "Recepção", que envolve planejamento, "Refeitório", que envolve flexibilidade mental e "Barraca", que envolve planejamento e flexibilidade mental. 
Já quando o subteste de reconhecimento de palavras da ADAS-Cog encontrava-se alterado, em associação ou não com a EDG, foram propostas as atividades denominadas "Travessia no Rio", "Baú do Tesouro", "Caverna" ou "Alojamento" e software Memo Training ${ }^{\circledR}$. Convém ressaltar que, no caso da ocorrência de depressão, o participante era encaminhado a um serviço de psicologia.

A cada sessão, o nível de complexidade das atividades foi aumentando gradualmente, respeitando a complexidade das tarefas em cada habilidade auditiva e/ou cognitiva trabalhada e considerando as dificuldades e as necessidades apresentadas pelos participantes.

Os resultados foram analisados estatisticamente, por meio da análise descritiva de todas as variáveis. Para realizar a comparação entre os testes comportamentais, ao considerar os dois momentos de avaliação em cada grupo, foi utilizado o teste de Wilcoxon para amostras relacionadas. Já para realizar a comparação entre os grupos, foi realizado o teste Mann Whitney para amostras independentes. A análise de correlação entre as variáveis foi feita por meio do coeficiente de correlação de Spearman. O pacote estatístico utilizado foi o Statistical Package for Social Sciences (SPSS), versão 17.0 e o nível de significância adotado foi de 5\%, ou seja, $\mathrm{p}<0,05$.

\section{RESULTADOS}

Participaram da amostra 25 (35,4\%) mulheres e 47 (64,6\%) homens, com idade mínima de 60 anos e máxima de 89 anos, média de 74 anos $( \pm 7,8)$, totalizando 72 idosos.

O grupo experimental foi constituído por $48(66,7 \%)$ idosos e o grupo controle por $24(33,3 \%)$ idosos. A média de idade do grupo experimental foi de 74 anos e 35 meses $( \pm 7,8)$, enquanto a média da mesma variável do grupo controle foi 72 anos e 4 meses $( \pm 6,09)$. Tal diferença não foi significativa $(p=0,205)$.
Dos 48 idosos do grupo experimental, 32 (66,7\%) eram homens e $16(33,3 \%)$, mulheres. Já o grupo controle foi constituído por 24 idosos, sendo $15(62,5 \%)$ homens e 9 (37,5\%), mulheres.

$\mathrm{O}$ desempenho nos testes comportamentais de ambos os grupos, nos dois momentos de avaliação, está demonstrado na Tabela 1.

Em relação ao desempenho no questionário de restrição de participação (HHIE-S), para o grupo experimental, na avaliação inicial, $6(12,5 \%)$ participantes apresentaram restrição de leve a moderada, $40(83,3 \%)$, restrição severa e apenas $2(4,2 \%)$ participantes não apresentaram restrição de participação. Após a realização do treinamento auditivo, $5(10,4 \%)$ participantes apresentaram restrição de leve a moderada, $1(2,1 \%)$ apresentou restrição severa e $42(87,5 \%)$ participantes não apresentaram restrição de participação. Para o grupo controle, os dados encontravam-se disponíveis para apenas 8 participantes, por questões externas ao controle dos pesquisadores. Os 8 participantes $(100 \%)$ apresentaram restrição de participação severa na pré-avaliação e ausência de restrição na pós-avaliação. $\mathrm{O}$ desempenho no referido questionário é demonstrado na Tabela 2.

Na sequência, realizou-se a análise comparativa das variáveis de interesse entre os grupos experimental e controle, por meio do coeficiente delta de cada variável, calculado pela fórmula: variável X (variável final - variável inicial) * 100/ variável inicial. Após a criação da nova variável, o comparativo entre os grupos foi realizado utilizando o teste Mann Whitney, como mostra a Tabela 3.

Em relação à análise comparativa das variáveis entre os gêneros, não foram observadas diferenças significativas para as variáveis de interesse.

As análises de correlação foram realizadas entre as variáveis. A Figura 1 mostra a correlação entre a idade e o desempenho no teste RGDT, do grupo experimental, ao comparar a avaliação pré-treinamento $(\mathrm{p}=0,039)$ e pós-treinamento auditivo $(\mathrm{p}=0,028)$.

Tabela 1. Desempenho nos testes comportamentais (média e desvio padrão) dos grupos experimental e controle na pré-avaliação e na pós-avaliação

\begin{tabular}{|c|c|c|c|c|c|c|c|c|c|c|}
\hline & \multicolumn{5}{|c|}{ Pré-avaliação } & \multicolumn{5}{|c|}{ Pós-avaliação } \\
\hline & $\begin{array}{c}\text { FR OD } \\
\%\end{array}$ & $\begin{array}{c}\text { FR OE } \\
\%\end{array}$ & $\begin{array}{c}\text { RGDT } \\
\text { ms }\end{array}$ & $\begin{array}{c}\text { DD OD } \\
\%\end{array}$ & $\begin{array}{c}\text { DD OE } \\
\%\end{array}$ & $\begin{array}{c}\text { FR OD } \\
\%\end{array}$ & $\begin{array}{c}\text { FR OE } \\
\%\end{array}$ & $\begin{array}{c}\text { RGDT } \\
\text { ms }\end{array}$ & $\begin{array}{c}\text { DD OD } \\
\%\end{array}$ & $\begin{array}{c}\text { DD OE } \\
\%\end{array}$ \\
\hline $\begin{array}{l}\text { Grupo } \\
\text { Experimental } \\
(n=48)\end{array}$ & $\begin{array}{c}28,00 \\
( \pm 16,1)\end{array}$ & $\begin{array}{c}27,66 \\
( \pm 17,1)\end{array}$ & $\begin{array}{c}78,47 \\
( \pm 34,5)\end{array}$ & $\begin{array}{c}45,88 \\
( \pm 14,5)\end{array}$ & $\begin{array}{c}50,78 \\
( \pm 14,4)\end{array}$ & $\begin{array}{c}48,58 \\
( \pm 17,9)\end{array}$ & $\begin{array}{c}48,41 \\
( \pm 18,2)\end{array}$ & $\begin{array}{c}49,66 \\
( \pm 24,7)\end{array}$ & $\begin{array}{c}65,62 \\
( \pm 15,2)\end{array}$ & $\begin{array}{r}68,90 \\
( \pm 14,5)\end{array}$ \\
\hline $\begin{array}{l}\text { Grupo } \\
\text { Controle } \\
(n=24)\end{array}$ & $\begin{array}{c}28,83 \\
( \pm 16,2)\end{array}$ & $\begin{array}{c}30,00 \\
( \pm 17,2)\end{array}$ & $\begin{array}{c}53,15 \\
( \pm 42,4)\end{array}$ & $\begin{array}{c}47,91 \\
( \pm 22,4)\end{array}$ & $\begin{array}{c}61,66 \\
( \pm 22,8)\end{array}$ & $\begin{array}{c}35,50 \\
( \pm 19,8)\end{array}$ & $\begin{array}{c}37,16 \\
( \pm 20,5)\end{array}$ & $\begin{array}{c}49,19 \\
( \pm 43,7)\end{array}$ & $\begin{array}{c}54,27 \\
( \pm 23,0)\end{array}$ & $\begin{array}{c}65,64 \\
( \pm 19,3)\end{array}$ \\
\hline \multicolumn{6}{|c|}{ Grupo Controle } & \multicolumn{5}{|c|}{ Grupo Experimental } \\
\hline Valor de $p$ & $0,002^{*}$ & $0,021^{*}$ & $0,005^{\star}$ & $0,004^{\star}$ & $0,060^{*}$ & $0,000^{*}$ & $0,000^{*}$ & $0,000^{\star}$ & $0,000^{*}$ & $0,000^{*}$ \\
\hline
\end{tabular}

${ }^{*}$ Teste Wilcoxon para amostras relacionadas

Legenda: FR = Fala no ruído; RGDT = Randon Gap Detection Test, DD = Dicótico de Dígitos; $\mathrm{OD}=$ orelha direita; $\mathrm{OE}=$ orelha esquerda; $\mathrm{ms}=$ milissegundos

Tabela 2. Desempenho no questionário de restrição de participação (média e desvio padrão) dos grupos experimental e controle na pré-avaliação e na pós-avaliação

\begin{tabular}{|c|c|c|}
\hline & Pré-avaliação & Pós-avaliação \\
\hline & HHIE-S & HHIE-S \\
\hline Grupo Experimental $(n=48)$ & $63,54( \pm 21,8)$ & $10,41( \pm 8,2)$ \\
\hline \multirow[t]{2}{*}{ Grupo Controle $(n=8)$} & $76,87( \pm 7,9)$ & $11,87( \pm 3,7)$ \\
\hline & Grupo Experimental & Grupo Controle \\
\hline Valor de $p$ & $0,000^{*}$ & $0,011^{*}$ \\
\hline
\end{tabular}

${ }^{*}$ Teste Wilcoxon para amostras relacionadas

Legenda: HHIE-S = Hearing Handicap Inventory for the Elderly- Screening 
Observou-se que o desempenho diminuiu (aumento do intervalo de gap em ms), à medida que a idade aumentava. Em relação ao grupo controle, identificou-se o mesmo resultado, ao observar os dados, porém, a análise não foi significativa, sendo a avaliação pré-treinamento $\mathrm{p}=0,50$ e pós-treinamento $\mathrm{p}=0,56$.

A Figura 2 apresenta a correlação entre o desempenho no teste de Fala no Ruído para a orelha direita e o teste Dicótico de Dígitos para a orelha direita, do grupo experimental, ao comparar a avaliação pré-treinamento $(\mathrm{p}=0,005)$ e pós-treinamento $(\mathrm{p}=0,001)$ auditivo. Observou-se correlação diretamente proporcional entre as variáveis, o que não foi observado nos resultados obtidos na orelha esquerda.

Ao realizar a análise de correlação entre o desempenho no questionário de restrição de participação (HHIE-S) e o desempenho nos testes comportamentais, não foram observadas relações significativas.

Tabela 3. Desempenho nos testes comportamentais e no questionário de restrição de participação (mediana) dos grupos experimental e controle

\begin{tabular}{lcccccc}
\hline \multirow{2}{*}{ Delta } & \multicolumn{3}{c}{ Grupo Experimental } & \multirow{2}{*}{ HHIE-S } \\
\cline { 2 - 6 } & FR OD & FR OE & RGDT & DD OD & DDOE \\
\hline Grupo Experimental (Mediana) & $83,3 \%$ & $79,1 \%$ & $-30,7 m$ & $34,0 \%$ & $38,6 \%$ & $-83,3 \%$ \\
Grupo Controle (Mediana) & $16,6 \%$ & $9,0 \%$ & $-9,0 m$ & $15,0 \%$ & $6,1 \%$ & $-82,8 \%$ \\
Valor de p & $\mathbf{0 , 0 0 0 *}$ & $\mathbf{0 , 0 0 0 *}$ & $\mathbf{0 , 0 0 0}^{*}$ & $\mathbf{0 , 0 0 2}$ & $\mathbf{0 , 0 0 0}^{*}$ & 0,991 \\
\hline
\end{tabular}

*Teste Mann Whitney para amostras independentes

Legenda: FR = Fala no ruído; RGDT = Randon Gap Detection Test, DD = Dicótico de Dígitos; HHIE-S = Hearing Handicap Inventory for the Elderly-Screening; $\mathrm{OD}=$ orelha direita; $\mathrm{OE}=$ orelha esquerda; $\mathrm{m}=$ milissegundos
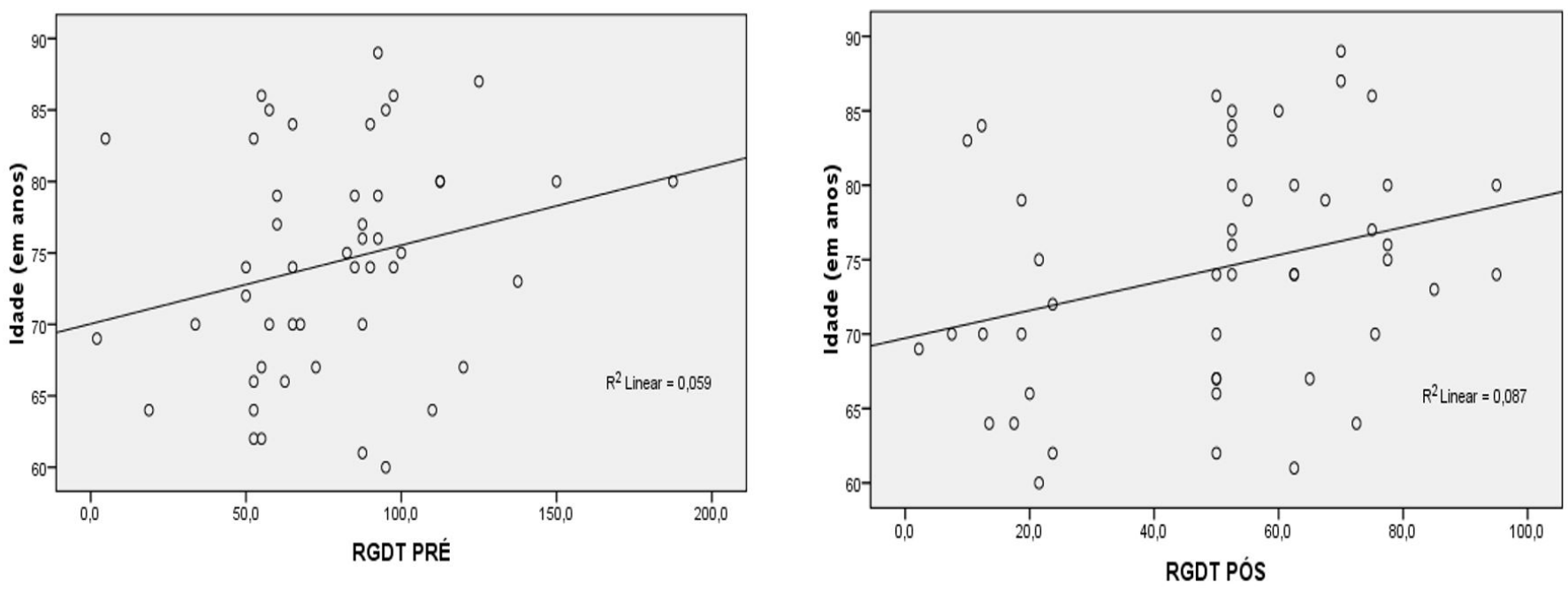

Figura 1. Correlação entre a idade e o desempenho no Random Gap Detection Test, do grupo experimental, ao comparar a avaliação pré-treinamento e pós-treinamento auditivo. Coeficiente de correlação de Spearman Legenda: RGDT = Randon Gap Detection Test
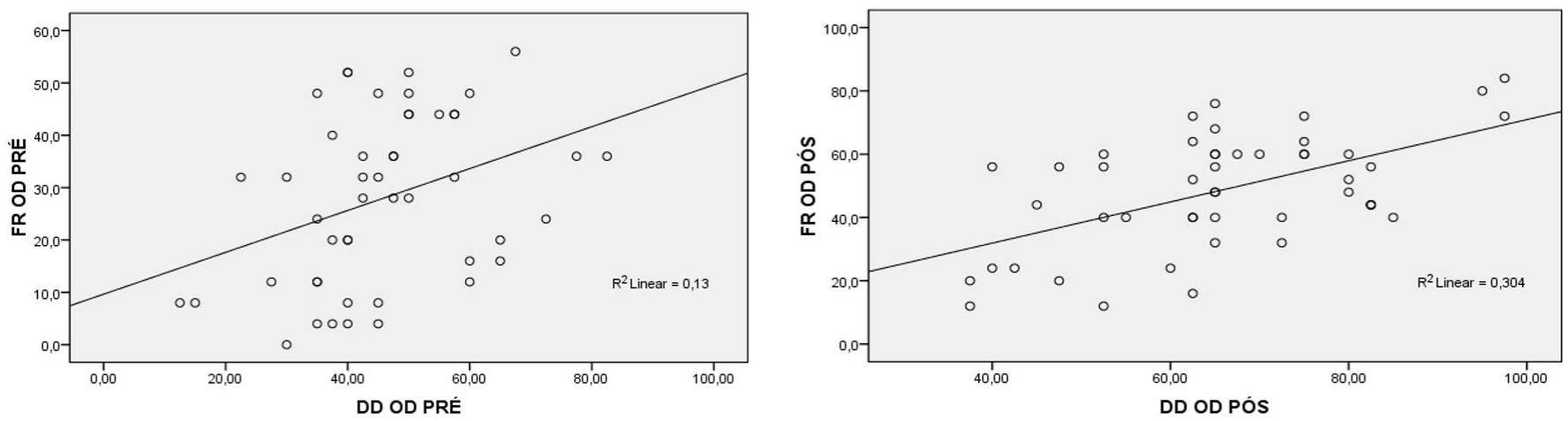

Figura 2. Correlação entre o desempenho no teste de Fala no Ruído para a orelha direita e o teste Dicótico de Dígitos para a orelha direita, do grupo experimental, ao comparar a avaliação pré-treinamento e pós-treinamento auditivo. Coeficiente de correlação de Spearman Legenda: FR = Fala no Ruído; $\mathrm{DD}=$ Dicótico de Dígitos; $\mathrm{OD}=$ Orelha Direita 


\section{DISCUSSÃO}

A pesquisa foi composta por 72 idosos divididos entre grupo experimental e controle, sendo 25 mulheres e 47 homens, com média de idade de 74 anos, semelhante a outros dois estudos realizados com a mesma amostragem, cujos idosos participantes foram submetidos a um programa de reabilitação auditiva, em que a amostra foi composta por 17 indivíduos, sendo seis mulheres e 11 homens, na faixa etária de 60 a $84 \operatorname{anos}^{(9,20)}$.

A amostra contou com um número maior de homens, porém, em outros estudos realizados com treinamento auditivo, as mulheres foram em maior número ${ }^{(21,22)}$. Este fato pode ser relacionado à grande procura das mulheres pelo diagnóstico fonoaudiológico precoce e pelo fato de buscarem mais por atendimentos em saúde do que os homens, concordando com outro estudo, que constatou menor participação dos homens nos serviços de atenção primária à saúde, no qual se observou que as práticas preventivas, por variados motivos, fossem elas de ordem estrutural e/ou cultural, não faziam parte do cotidiano da população masculina ${ }^{(23)}$. Em contrapartida, uma pesquisa relatou maior incidência de perda auditiva nos indivíduos do sexo masculino, pois estes, possivelmente, já apresentavam um declínio da acuidade auditiva com a idade, como fator de risco independente para perda auditiva. Além disso, encontravam-se mais expostos do que as mulheres a fatores e causas ambientais, como a exposição a ruídos ocupacionais e ingestão de bebidas alcoólicas ${ }^{(2)}$. Tais fatores poderiam justificar a maior procura de homens pelo atendimento proporcionado pelo centro de saúde auditiva, uma vez que a amostra da presente pesquisa não foi pareada.

O grupo experimental foi composto por 48 idosos e o grupo controle por 24, pois foi constituído pelos participantes que não desejaram realizar o treinamento auditivo, por dificuldades em relação ao deslocamento.

Quanto aos resultados da segunda avaliação do processamento auditivo, houve relação significativa para ambos os grupos, referindo-se ao uso do AASI para o grupo controle e ao uso do AASI associado à terapia fonoaudiológica, para o grupo experimental. O mesmo resultado foi encontrado em um estudo que avaliou os efeitos da reabilitação auditiva, por meio da análise dos aspectos quantitativos e qualitativos do Teste Dicótico de Dissílabos Alternados (SSW), em idosos novos usuários de próteses auditivas ${ }^{(20)}$. Em outro estudo, o desempenho de idosos nos testes comportamentais foi avaliado na entrega do AASI, após um mês de uso do dispositivo e após o treinamento auditivo. Observou-se que todos os participantes aumentaram os escores nos testes comportamentais, gradativamente, obtendo melhor desempenho após o treinamento auditivo no segundo momento da avaliação, sugerindo que, tanto o processo de adaptação do AASI, quanto o treinamento auditivo, auxiliaram na melhora das habilidades auditivas alteradas ${ }^{(21)}$.

No que se refere ao desempenho no questionário HHIE-S, os resultados mostraram diferenças estatisticamente significativas, tanto para o grupo experimental, quanto para o grupo controle (oito participantes), qualitativamente superiores aos testes de processamento, o que indica que o uso do AASI contribuiu para que ouvissem melhor, diminuindo consideravelmente a restrição de participação. Uma pesquisa aplicou o mesmo questionário com 29 idosos, antes da utilização do AASI e após um ano de uso e constatou-se redução significativa da restrição de participação após o uso da prótese auditiva, no grupo estudado $^{(24)}$. Outro estudo, também semelhante, demonstrou que os idosos apresentaram redução da autopercepção das restrições de participação após o uso do AASI, atribuindo tal melhora à adaptação e ao uso das próteses auditivas ${ }^{(25)}$.

$\mathrm{Na}$ análise comparativa das variáveis de interesse, realizada por meio do coeficiente delta, examinou-se o quanto cada grupo melhorou, considerando a mediana, que, de certa forma, desconsiderou aqueles participantes que não obtiveram melhora e aqueles cuja melhora foi bem acima da média. A partir desse coeficiente, aplicou-se o teste de Mann Whitney, por meio do qual se constatou que o grupo experimental melhorou superiormente ao grupo controle, no segundo momento da avaliação, sendo constatadas diferenças estatisticamente significativas para todos os testes comportamentais, exceto para o questionário de restrição de participação. Este mesmo resultado também foi encontrado numa pesquisa realizada com o objetivo de verificar os efeitos do treinamento auditivo, em que apenas o grupo experimental dos indivíduos com comprometimento cognitivo obteve resultados estatisticamente significativos para os testes comportamentais, no segundo momento da avaliação $^{(22)}$. Em outro estudo, também realizado com idosos, observaram-se efeitos da reabilitação auditiva na habilidade de ordenação temporal e verificou-se que o desempenho do grupo experimental apresentou evolução, quando considerados os resultados obtidos antes e após o treinamento auditivo, sendo que o mesmo resultado não foi observado no grupo controle ${ }^{(9)}$.

Observou-se que o desempenho obtido nos testes RGDT e Dicótico de Dígitos (redução e aumento, respectivamente, em torno de $30 \%$ a $38 \%$ ), no segundo momento da avaliação, não melhorou da mesma forma que o desempenho obtido no teste Fala no Ruído (aumento em torno de $80 \%$, para ambas as orelhas), como apresentado na Tabela 3. Este fato pode estar relacionado à cognição que, no idoso, é caracterizada pela lentidão, sugerindo deficit na transmissão do processamento temporal, bem como à redução da eficiência do corpo caloso. A mesma constatação foi observada em relação ao desempenho nesses testes, ao analisar os resultados obtidos em outro estudo ${ }^{(21)}$.

Conforme proposto pela Portaria SAS/MS n ${ }^{\circ} 587^{(19)}$, são ofertadas quatro sessões de terapia fonoaudiológica para a população adulta com deficiência auditiva. Um estudo de revisão sistemática concluiu que o processo de reabilitação poderia constar de, no mínimo, oito sessões realizadas semanalmente, com a duração de 45 minutos a uma hora ${ }^{(26)}$.

$\mathrm{O}$ estudo supracitado constatou que um programa de treinamento auditivo eficaz poderia envolver tarefas de interação binaural, fechamento auditivo e figura-fundo, resolução temporal, ordenação temporal e estímulos dicóticos, associadas às funções cognitivas de atenção, linguagem, memória e funções executivas. A presente pesquisa contou com um treinamento auditivo computadorizado, constituído de um conjunto de condições e/ou tarefas acústicas designadas para ativar o sistema auditivo e sistemas relacionados, de tal maneira que os comportamentos auditivos associados e sua base neural fossem alterados de maneira positiva. $\mathrm{O}$ programa incluiu atividades para a estimulação de todas as habilidades acima citadas pelo estudo, realizadas por meio de softwares que, segundo a literatura estudada, oferecem importantes ganhos na percepção auditiva após o treinamento e, além disso, atraem e mantêm a atenção dos usuários, propiciando condições cognitivas que favorecem a aprendizagem ${ }^{(27)}$. Além disso, o programa focou, prioritariamente, as funções nas quais os participantes apresentaram maior dificuldade, o que reforça o entendimento 
de que os resultados poderiam ter sido melhores, se houvesse maior número de sessões.

$\mathrm{O}$ treinamento auditivo computadorizado foi realizado por meio de softwares e computadores fora da cabine acústica. A literatura destacou que o treinamento auditivo neurocognitivo pode ser complementar ao treinamento auditivo formal, constituindo-se em prática intensiva e extensiva, que inclui o treinamento das funções cognitivas, maximizando a generalização e a efetividade do tratamento(28).

Ainda por meio da análise realizada com o coeficiente delta, observou-se que os dois grupos obtiveram melhora na restrição de participação, não sendo observadas diferenças estatísticas significativas, sugerindo que a melhora dos resultados obtidos por meio do questionário, nesta pesquisa, relacionou-se mais com o uso do AASI do que com a terapia fonoaudiológica, propriamente dita, o que confirma os resultados de outro estudo, em que a restrição de participação, investigada por meio do questionário HHIE-S, foi reduzida apenas com o uso efetivo do $\mathrm{AASI}^{(29)}$.

Em relação à análise comparativa das variáveis entre os gêneros, não foram observadas diferenças significativas para as variáveis de interesse, concordando com outros estudos encontrados na literatura pesquisada ${ }^{(20,21)}$.

Ao relacionar a idade e o desempenho no teste RGDT, do grupo experimental, pré-treinamento e pós-treinamento auditivo, observou-se que o desempenho diminuiu à medida que a idade aumentava. A mesma constatação foi verificada no grupo controle (notou-se que os participantes mais velhos apresentaram maiores intervalos de tempo), porém, a análise de correlação não foi significativa, talvez em função do número menor de participantes. O objetivo desta análise foi atentar para o processo de envelhecimento, que traz consigo o declínio na função auditiva central. Assim, são vários os fenômenos comportamentais envolvidos nesta função. Entre eles, encontram-se a resolução temporal e a ordenação temporal. Um estudo mostrou que o processo de envelhecimento causa deterioração no processamento temporal, que pode estar associado ao decréscimo da velocidade de processamento no córtex auditivo primário ${ }^{(29)}$. Em um estudo que avaliou a percepção auditiva de ordem temporal em centenários, os resultados obtidos confirmaram a redução da resolução temporal relacionada com a idade e atestaram que, quanto maior a idade, maior a alteração, o que pode ser explicado pela desaceleração do processamento da informação, comumente postulada como o mais antigo e distinto sintoma do envelhecimento cognitivo ${ }^{(30)}$.

Referente à análise de correlação entre o desempenho no teste Fala no Ruído e o teste Dicótico de Dígitos, ambos para a orelha direita, no pré-treinamento e pós-treinamento auditivo do grupo experimental, observou-se correlação diretamente proporcional. O teste Dicótico de Dígitos foi realizado somente na etapa denominada integração binaural, que avalia a habilidade de processar a mensagem auditiva apresentada em ambas as orelhas, e requer a integridade do corpo caloso, proporcionando a integração intra-hemisférica e inter-hemisférica. $\mathrm{O}$ teste Fala no Ruído avalia a habilidade de fechamento auditivo e necessita da integridade de todo o tronco encefálico. Apesar de ambos os testes não medirem a mesma habilidade, são relevantes para discriminação.

No que diz respeito à análise de correlação entre o questionário HHIE-S e os testes de processamento auditivo, não foram observadas relações significativas, pois são medidas distintas. O HHIE-S é uma medida subjetiva ${ }^{(23)}$, enquanto que os testes de processamento auditivo, que também se constituem em medidas subjetivas, avaliam o funcionamento das vias auditivas do sistema nervoso central e/ou das áreas integrativas, mensuradas pelo avaliador, por meio da aplicação de testes. Cabe ressaltar que, apesar de não medirem o mesmo construto, podem ser medidas complementares do processo de reabilitação.

Por fim, a melhora proporcionada pelo treinamento auditivo neurocognitivo nas distintas habilidades auditivas avaliadas está relacionada, muito provavelmente, à capacidade do sistema nervoso auditivo central de mudar frente à estimulação ambiental e esta capacidade pode ser definida como plasticidade neural. Desta forma, pode-se afirmar que o programa de treinamento auditivo computadorizado, utilizado no presente estudo, induziu às mudanças benéficas no sistema nervoso central nos idosos, principalmente do grupo experimental, mudanças estas que puderam ser confirmadas pela melhora no desempenho dos sujeitos nos testes Dicótico de Dígitos, RGDT e Fala no Ruído.

Ainda assim, é importante que mais pesquisas com número similar de indivíduos nos grupos experimental e controle, incluindo um grupo placebo e com um número maior de sessões que envolvam o treinamento das habilidades auditivas na modalidade formal e/ou informal em idosos. Deste modo, poderão oferecer maior ganho na qualidade da comunicação desses indivíduos e acrescentar informações importantes sobre a influência dessas habilidades na percepção da fala, sendo que a melhora da condição auditiva trará maiores benefícios para o idoso e para a sociedade.

Cabe ressaltar, também, a importância de integrar avaliações cognitivas e eletrofisiológicas em pesquisas semelhantes, para comprovar os resultados e expandir tais conhecimentos aos serviços de saúde.

\section{CONCLUSÃO}

O programa de treinamento auditivo computadorizado neurocognitivo se mostrou eficaz, indicando melhora nas habilidades auditivas alteradas, observadas por meio dos testes comportamentais, com apenas quatro sessões. O questionário de restrição de participação não refletiu os efeitos do treinamento auditivo.

Evidencia-se a relevância e o impacto deste trabalho para os serviços de saúde auditiva, reforçando a utilização de medidas cognitivas associadas ao processamento auditivo nesses serviços. Porém, tendo em vista que as verbas são custeadas pelo Sistema Único de Saúde, é necessária a organização de um protocolo mais reduzido.

\section{REFERÊNCIAS}

1. Mesquita LG, Pereira LD. Processamento temporal em idosos: o efeito da habilidade de resolução temporal em tarefas de ordenação de série de sons. Rev CEFAC. 2013;15(5):1163-9. http://dx.doi.org/10.1590/ S1516-18462013005000038.

2. Martins K, Fontenele M, Câmara S, Sartorato EL. Genetic and audiologic study in elderly with sensorineural hearing loss. CoDAS. 2013;25(3):224-8. http://dx.doi.org/10.1590/S2317-17822013000300006. PMid:24408332. 
3. Moda I, Mantello EB, Reis ACMB, Isaac ML, Oliveira AA, Hyppolito MA. Avaliação da satisfação do usuário de aparelho de amplificação sonora. Rev CEFAC. 2013;15(4):778-85. http://dx.doi.org/10.1590/ S1516-18462013000400006.

4. Mondelli MFCG, Souza PJS. Quality of life in elderly adults before and after hearing aid fitting. Braz J Otorhinolaryngol. 2012;78(3):49-56. http://dx.doi.org/10.1590/S1808-86942012000300010. PMid:22714847.

5. Rodrigues CCC, Aurélio FS, Silva VB, Lopes TA. Benefício fornecido pelo uso de aparelhos de amplificação sonora individual em idosos de um programa de saúde auditiva de Porto Velho - RO. Rev CEFAC. 2013;15(5):1170-80. http://dx.doi.org/10.1590/S151618462013005000025 .

6. Buss LH, Rossi AG, Buss CH, Oliveira RC. Desempenho nas habilidades auditivas de atenção seletiva e memória auditiva em um grupo de idosos protetizados: influência de perda auditiva, idade e gênero. Rev CEFAC. 2013;15(5):1065-72. http://dx.doi.org/10.1590/ S1516-18462012005000100.

7. Buss LH, Buss CH, Backes CC, Oliveira RC. Achados no teste SSW em um grupo de idosos usuários de próteses auditivas pós-período de aclimatização. Cient Ciênc Biol Saúde. 2014;16(1):33-7. http:// dx.doi.org/10.17921/2447-8938.2014v16n1p\%25p.

8. Ramos BD. But, after all, why is it important to assess the auditory processing? Braz J Otorhinolaryngol. 2013;79(5):529. http://dx.doi. org/10.5935/1808-8694.20130097. PMid:24141665.

9. Hennig TR, Costa MJ, Rossi AG, Moraes AB. Efeitos da reabilitação auditiva na habilidade de ordenação temporal em idosos usuários de próteses auditivas. Rev Soc Bras Fonoaudiol. 2012;24(1):26-33. http:// dx.doi.org/10.1590/S2179-64912012000100006.

10. Lacerda C, Silva L, Canto RT, Cheik N. Effects of hearing AIDS in the balance, quality of life and fear to fall in elderly people with sensorineural hearing loss. Int Arch Otorhinolaryngol. 2012;16(2):156-62. http:// dx.doi.org/10.7162/S1809-97772012000200002. PMid:25991930.

11. Irigaray TQ, Gomes I Fo, Schneider RH. Efeitos de um treino de atenção, memória e funções executivas na cognição de idosos saudáveis. Psicol Reflex Crit. 2012;25(1):188-202. http://dx.doi.org/10.1590/ S0102-79722012000100023.

12. Cruz ACA, Andrade AN, Gil D. A eficácia do treinamento auditivo formal em adultos com distúrbio do processamento auditivo (central). Rev CEFAC. 2013;15(6):1427-34. http://dx.doi.org/10.1590/S151618462013000600004 .

13. Silva MP, Comerlatto AA Jr, Balen SA, Bevilacqua MC. O uso de um software na (re)habilitação de crianças com deficiência auditiva. J Soc Bras Fonoaudiol. 2012;24(1):34-41. http://dx.doi.org/10.1590/ S2179-64912012000100007. PMid:22460370.

14. Conselho Federal de Fonoaudiologia. Manual de procedimentos em audiometria tonal liminar, logoaudiometria e medidas de imitância acústica. Brasília: CFFa; 2013.

15. Lloyd LL, Kaplan H. Audiometric interpretation: a manual o basic audiometry. Baltimore: University Park Press; 1978.

16. Menegotto IH, Soldera CLC, Anderle P, Anhaia TC. Correlation between hearing loss and the results of the following questionnaires: Hearing
Handicap Inventory for the Adults - Screening Version HHIA-S and Hearing Handicap Inventory for the Elderly - Screening Version HHIE-S. Arq Int Otorrinolaringol. 2011;15(3):319-26. http://dx.doi. org/10.1590/S1809-48722011000300009.

17. Pereira LD, Schochat E. Testes auditivos comportamentais para avaliação do processamento auditivo central. Barueri: Pró-fono; 2011.

18. Costa LP, Pereira LD, Santos MFC. Auditory fusion test in scholars. Pro Fono. 2004;16(2):187-96. PMid:15311743.

19. Brasil. Ministério da Saúde. Portaria n ${ }^{\circ} 587$, de 7 de outubro de 2004. Redes estaduais de atenção à saúde auditiva. Diário Oficial da União [Internet]; Brasília; 2004 [citado em 5 Jul 2015]. Disponível em: http:// www.saude.mg.gov.br/images/documentos/Portaria_587.pdf

20. Lessa AH, Hennig TR, Costa MJ, Rossi AG. Resultados da reabilitação auditiva em idosos usuários de próteses auditivas avaliados com teste dicótico. CoDAS. 2013;25(2):169-75. http://dx.doi.org/10.1590/ S2317-17822013000200013. PMid:24408247.

21. Fonseca GCR, Costa-Ferreira MID. O desempenho de idosos com perda auditiva neurossensorial nos testes de processamento auditivo: um estudo longitudinal. Rev CEFAC. 2015;17(3):809-18. http://dx.doi. org/10.1590/1982-0216201513114.

22. Ávila RRA, Murphy CFB, Schochat E. Efeitos do treinamento auditivo em idosos com comprometimento cognitivo leve. Psicol Reflex Crit. 2013;27(3):547-55. http://dx.doi.org/10.1590/1678-7153.201427316.

23. Alves RF, Silva RP, Ernesto MV, Lima AGB, Souza FM. Gênero e saúde: o cuidar do homem em debate. Psicol Teor Prat. 2011;13(3):15266.

24. Guarinello AC, Marcelos SB, Ribas A, Marques JM. Análise da percepção de um grupo de idosos a respeito de seu handicap auditivo antes e após o uso do aparelho auditivo. Rev Bras Geriatr Gerontol. 2013;16(4):739-45. http://dx.doi.org/10.1590/S1809-98232013000400008.

25. Magalhães R, Iório MCM. Quality of life and participation restrictions, a study in eldery. Braz J Otorhinolaryngol. 2011;77(5):628-38. http:// dx.doi.org/10.1590/S1808-86942011000500016. PMid:22030973.

26. Beier LO, Pedroso F, Costa-Ferreira MID. Benefícios do treinamento auditivo em usuários de aparelho de amplificação sonora individual: revisão sistemática. Rev CEFAC. 2015;17(4):1327-32. http://dx.doi. org/10.1590/1982-0216201517422614.

27. Alvarez AMM. Processamento auditivo: treinamento auditivo neurocognitivo. In: Bevilacqua MC, Martinez MAN, Balen SA, Pupo AC, Reis ACMB, Frota S, editores. Tratado de audiologia. São Paulo: Santos; 2014. p. 845-59.

28. Dias KZ. Treino auditivo formal nos distúrbios de processamento auditivo. In: Bevilacqua MC, Martinez MAN, Balen SA, Pupo AC, Reis ACMB, Frota S, editores. Tratado de audiologia. São Paulo: Santos; 2014. p. 829-44.

29. Matos GGO, Frota S. Resolução temporal em perdas auditivas sensorioneurais. Audiol Commun Res. 2013;18(1):30-6. http://dx.doi. org/10.1590/S2317-64312013000100007.

30. Kołodziejczyk I, Szelsg E. Auditory perception of temporal order in centenarians in comparison with young and elderly subjects. Acta Neurobiol Exp. 2008;68(3):373-81. PMid:18668160. 